American Journal of Geoscience 1 (1): 7-11, 2010

ISSN 1948-9846

(C) 2010 Science Publications

\title{
Assessing the Applicability of Landslide Databases for Hazard Management Using Objective and Subjective Models: Australian Geoinformatic Case
}

\author{
M. Ownegh \\ Department of Arid Zone Management, Gorgan University of Agricultural, \\ Sciences and Natural Resources, Gorgan, Iran
}

\begin{abstract}
Problem statement: In order to assess the applicability of Australian landslide databases as a hazard management support system, current hard and soft literature and data sources were screened. This assessment resulted in the selection of four main data bases including national landslide database (A), Peril Aus II (B), the Cities project (C) and analytical study of Fell (D). Approach: In this study applicability of databases was evaluated using two different methods: Numerical model (objective) and AHP model (subjective). After statistical analysis, the results of this evaluation were combined. Due to shortage of a definite standard, a simple numerical model has been developed with 4 main complex parameters (each one contains 4 minor parameters). This includes: Graphical-statistics, geo-spatial, physico-temporal and techno-management, value scale of $0-3$ and 4 applicability classes and relative importance of the databases has been evaluated. Relative priority of the databases as competitor alternatives was determined using Analytical Hierarchy Process (AHP) technique as a math-logical tool for decision making in uncertainty, by expert based pair-wise comparison $(\mathrm{CR}=0.0296)$. Finally its value was normalized to the scale of numerical model for comparison. Results: According to the results, in both models applicability classes of databases range from II-IV with only one class difference. With merging numerical outputs of two models by a $80 \%$ rank correlation in a single paradigm, the applicability class improve from II-III only in database A, but for others remain constant. Rank correlation between databases in different levels imply to different relationships, so that some of them such as $R_{A B}, R_{A C}, R_{A D}$ and $R_{B C}$ can be explained with the inductive theorems of genetically statistic-thematic multi-relations of databases. The differences of strengths and weaknesses of the Australian four main landslide databases that mainly affect their practical applicability for hazard management are considerable in spite of genetic relation and data overlaps. Thus, practical applicability of each database can be characterized well by two contrast objective and subjective models. Conclusion: As a result, applicability of Australian landslide databases is of class III (high). Thus, it still needs further development and complementary actions especially in geo-technical, geometric, impact (damage) data and map scale.
\end{abstract}

Key words: Landslide database, applicability class, numerical model, Analytical Hierarchy Process (AHP), hazard management

\section{INTRODUCTION}

Developing digital and spatial data bases of natural hazards (CSIRO, 2003) and easy access of data users, is of vital role for hazard management and reduction of natural tax in land use planning and sustainable development (NHRC, 1999; Ownegh, 2002a). In recent decades, some countries such as Australia and Canada have established national databases of natural hazards as land sliding and applied as a spatial decision support system in the management of environment and society both in regional and national scale (Geological survey of Canada, 1999). The new wave of this trend is globalization of natural hazards digital databases for organized and online data service for wide spectrum of data users with different objectives.

Trends in setup of long-term landslide databases by reconstruction of historical document and event records (as Australian and Canadian national databases along more than 160 years period) represent the necessity of historical and statistical approaches in landslide hazard behavior analysis. This is used for forecasting landslide hazard behavior based on principle of uniformity (Ken et al., 1999; Andrew et al., 1995; Geological survey of Canada, 1999) (Fig. 1).

From the endemic problems of landslide database developing in Australia can be implied to vast and continental area, absence of population in large central, 
Am. J. Geoscience 1 (1): 7-11, 2010

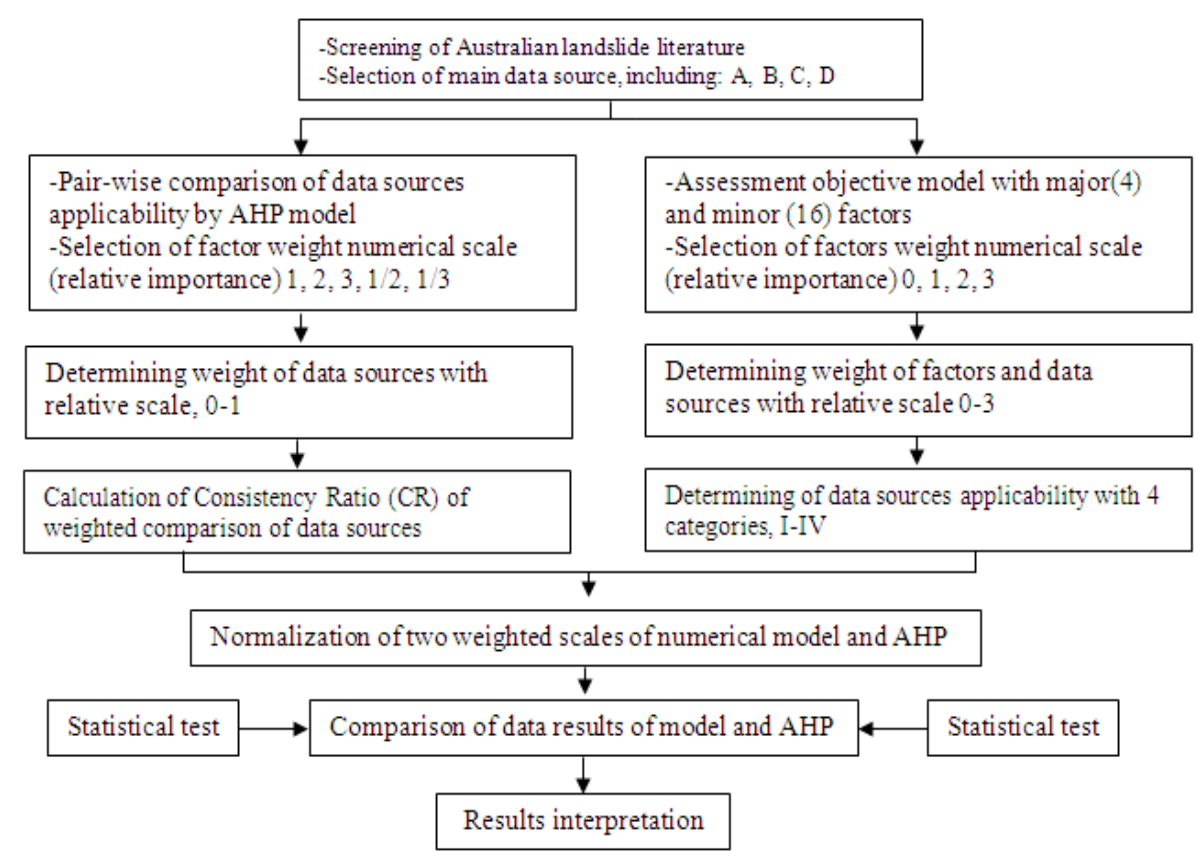

Fig. 1: Stages of assessing the Australian landslide databases

northern and western parts, lack of landowners cooperation, non-recovery of damage insurance, close spatial and temporal overlay of land sliding and other triggering hazards especially earthquake, cyclonic storm and flooding; the problem of real hazard and risk estimation and presentation of landslide management program (AGSO, 2001; Fell, 1995; Geoscience Australia, 2002; NHRC, 1999; 2000).

The main purpose of this study is to evaluate applicability of Australian landslide databases in landslide hazard and risk management with merging of two objective and subjective models (Ownegh, 2002b).

\section{MATERIALS AND METHODS}

At present due to the difference in landslide databases structure and variety of their users, there is not any special standard for assessing landslide database applicability from the point of hazard management and land use planning (Ownegh, 2002c).

A logical method for this kind of assessment is matching of recorded and estimated amounts of physical, geometrical and socio-economic key factors expected from event and management necessities of landslide. This practice needs an objective numerical model, supporting with Analytical Hierarchy Process (AHP, subjective model) and merging of their data results (Bontayan and Bishop, 1998; Ownegh, 2008; Saaty, 1980). For reduction of text, the successive stages of this research are summarized in flowchart (Fig. 1).

\section{RESULTS}

Due to variety, the results of this research were presented in separation of numerical model, AHP technique and their combination as follow:

Numerical value of key parameters varies between zero (none), 1 (low, class I) to 3 (high, very high class IV) in A and D and 1-3 in B and C databases. Average value of parameters for all databases varies from 1.5-3. Least SD and CV of values (both are zero) belong to cause parameter and most of them (1.24 and 86\%) to geotechnical data and technico-management comments parameters (Table 1). Average value of databases varies between 1.5 (in A) and 2.75 (in C). Their applicability classes are II in A, III in D and IV in B and C total average value of all databases is 2.2 and class II (Table 1). Difference of parameter frequency between numerical value classes is relatively high and varies from 0-13 in C. Parameter density in value 3(high, very high class IV) is considerable. These values are 4, 9, 13 and 6 in A to D respectively (Table 2). In total average of databases, difference of parameter frequency between applicability classes is very high and its mode lies in class III (Table 3). According to statistical test $(\mathrm{Ho}=$ no difference in repeated measurement), 
Am. J. Geoscience 1 (1): 7-11, 2010

Table 1: Weight value of factor relative importance for landslide databases by model

\begin{tabular}{|c|c|c|c|c|c|c|c|c|c|c|}
\hline Main parameter* & Databases** parameter & A & B & $\mathrm{C}$ & $\mathrm{D}$ & Sum & Ave & Class**** & SD & $\mathrm{CV}(\%)$ \\
\hline \multirow[t]{4}{*}{ Graphico-statistical } & Lineage & 1.0 & 2.0 & 3.00 & 3.00 & 9.0 & 2.25 & III & 0.96 & 42.49 \\
\hline & Data format & 3.0 & 3.0 & 3.00 & 1.00 & 10.0 & 2.50 & IV & 1.00 & 40.00 \\
\hline & Analysis method & 1.0 & 3.0 & 3.00 & 2.00 & 9.0 & 2.25 & III & 1.06 & 47.75 \\
\hline & Informative level & 1.0 & 2.0 & 2.00 & 3.00 & 8.0 & 2.00 & III & 0.81 & 40.82 \\
\hline \multirow{4}{*}{ Geo-spatial } & Geometric dimension & 1.0 & 2.0 & 3.00 & 3.00 & 9.0 & 2.25 & III & 0.90 & 40.08 \\
\hline & Type (class) & 2.0 & 2.0 & 3.00 & 3.00 & 10.0 & 2.50 & IV & 0.58 & 23.08 \\
\hline & Positional accuracy & 2.0 & 2.0 & 3.00 & 2.00 & 9.0 & 2.25 & III & 0.50 & 22.22 \\
\hline & Spatial coverage & 3.0 & 3.0 & 1.00 & 2.00 & 9.0 & 2.25 & III & 0.96 & 42.49 \\
\hline \multirow[t]{4}{*}{ Physico-temporal } & Statistical time period & 3.0 & 3.0 & 3.00 & 2.00 & 11.0 & 2.75 & IV & 0.50 & 18.18 \\
\hline & Cause & 3.0 & 3.0 & 3.00 & 3.00 & 12.0 & 3.00 & IV & 0.00 & 0.00 \\
\hline & Velocity/intensity & 2.0 & 3.0 & 3.00 & 2.00 & 10.0 & 2.50 & IV & 0.28 & 11.13 \\
\hline & Geotechnical data & 0.0 & 1.0 & 2.00 & 3.00 & 6.0 & 1.50 & II & 1.29 & 86.06 \\
\hline \multirow[t]{9}{*}{ Technico-managemental } & Sustained damage & 2.0 & 3.0 & 3.00 & 0.00 & 8.0 & 2.00 & III & 1.22 & 61.23 \\
\hline & Potential risk (probable) & 0.0 & 3.0 & 3.00 & 1.00 & 7.0 & 1.75 & III & 1.50 & 85.56 \\
\hline & Potential hazard (repeat) & 0.0 & 3.0 & 3.00 & 2.00 & 8.0 & 2.00 & III & 1.22 & 61.23 \\
\hline & Technico-manage.Comments & 0.0 & 2.0 & 3.00 & 1.00 & 6.0 & 1.50 & II & 1.29 & 86.06 \\
\hline & Sum & 23.0 & 40.0 & 44.00 & 33.00 & 141.0 & 2.20 & III & & \\
\hline & Ave & 1.5 & 2.5 & 2.75 & 2.06 & 2.2 & & & & \\
\hline & Class & II & IV & IV & III & III & & & & \\
\hline & SD & 1.15 & 0.63 & 0.50 & 0.76 & 0.76 & & & & \\
\hline & CV $(\%)$ & 76.98 & 25.29 & 18.76 & 36.94 & 39.49 & & & & \\
\hline
\end{tabular}

$\mathrm{Rsm}=0.1968$ between all rows indicate to relatively differences among parameters (rows) and databases; $*$ : Main parameters are classified as: Graphico-statistical, Geo--spatial, Physico-temporal and Technico-managemental. **: A = National landslide database, B = Peril Aus II, C= Cities project and $\mathrm{D}=$ Analytical paper of Fell; ***: Value scale: None $=0$, Low $=1$, Medium $=2$, High $=3$; ****: Applicability classes: Low I $(<0.75)$, medium II (0.76--1.5), high III (1.51--2.25), very high IV (2.26--3)

Table 2: Comparison of parameter numerical value for landslide databases

\begin{tabular}{lllll}
\hline & 0 (None) & 1 (Low) & 2 (Medium) & 3 (High) \\
\hline A & $12-14-15-16$ & $1-3-4-5$ & $6-7-11-13$ & $2-8-9-10$ \\
$\mathrm{~B}$ & - & 12 & $1-4-5-6-7-16$ & $2-3-8-9-10-11-13-14-15$ \\
$\mathrm{C}$ & - & 8 & $4-12$ & $1-2-3-5-6-7-9-10-11-13-14-15-16$ \\
$\mathrm{D}$ & 13 & $2-14-16$ & $3-7-8-9-11-15$ & $1-4-5-6-10-12$ \\
\hline
\end{tabular}

Table 3: Comparison of parameters applicability class for landslide databases (average for country)

\begin{tabular}{lllllr}
\hline Class & I & II & III & IV & Sum \\
\hline No. of para & - & $12-16$ & $1-3-4-5-7-8-13-14-15$ & $2-6-9-10-11$ & 16 \\
Percentage & - & 12.50 & 56.25 & 31.25 & 100 \\
\hline
\end{tabular}

there is a high confidence level $(\mathrm{p}=0.0078)$ that difference of observation (expert judgment) is not random. It implies on high thematic resolution of model and inherent difference of databases applicability also, (columns) (Table 1).

In AHP technique, the priority of landslide databases was calculated by very high consistency rate $(C R=0.0296)$ that is more accurate than the result of run out project $(\mathrm{CR}=0.0300)$ (Barredo et al., 2002).

Difference in applicability of relative weight of database is very high and varies between 0.1256 in $\mathrm{D}$ and 0.3320 in C. Applicability priority or ranks of C, B, $\mathrm{A}$ and $\mathrm{D}$ are $1-4$ respectively (Table 4 ).

By converting weight value of AHP to numerical model value scale (indeed merging and calculation of weight value ratio from sum value of relative importance in numerical model, 8.81), equivalency of partial and total (average) values of two models is possible.
Table 4: Comparison of model and AHP value for landslide databases

\begin{tabular}{lllllll}
\hline & A & B & C & D & Wi & Rank* \\
\hline A & 0.2222 & 0.3003 & 0.1666 & 0.2501 & 0.2348 & 3 \\
B & 0.2222 & 0.3003 & 0.3333 & 0.3751 & 0.3077 & 2 \\
C & 0.4444 & 0.3003 & 0.3333 & 0.2501 & 0.3320 & 1 \\
D & 0.1111 & 0.1001 & 0.1666 & 0.1251 & 0.1257 & 4 \\
Sum & 1.0000 & 1.0000 & 1.0000 & 1.0000 & 1.0000 & \\
\hline
\end{tabular}

*: Rank of weight or applicability

Table 5: Matrix of normalized coefficient of landslide databases

\begin{tabular}{lllllllll}
\hline & A & B & C & D & Ava & Class & SD & CV (\%) \\
\hline Model & 1.5 & 2.5 & 2.75 & 2.06 & 2.2 & III & 0.8797 & 39.49 \\
Class & II & IV & IV & III & - & III & - & - \\
AHP & 2.07 & 2.71 & 2.92 & 1.11 & 2.2 & III & 0.7874 & 35.77 \\
Class & III & IV & IV & II & - & III & - & - \\
Ava & 1.785 & 2.605 & 2.82 & 1.585 & 2.2 & III & 0.8335 & 37.63 \\
Class & III & IV & IV & III & - & III & - & - \\
d (\%) & +38 & +8.4 & +6.18 & -46.11 & 0 & 0 & 10.49 & 9.44 \\
\hline & & & & & & & &
\end{tabular}

According to statistical test, there is not a high confidence level $(\mathrm{p}<0.1414)$ on the merging of numerical model $(\mathrm{SD}=0.8797$ and $\mathrm{CV}=39.49)$ and AHP (SD $=0.7874$ and $\mathrm{CV}=35.77$ ) (each one was assumed as a replication or judgment) and rejection of Ho (no difference between applicability average ranks of databases in two model). However, considering the high coefficient of correlation $(\mathrm{r}=0.8, \mathrm{z}=1.3856$ and $\mathrm{p}=0.0829$ ), their results are combinable (Nie et al., 2001; Saaty, 1980). When combined, applicability class in database A increases from II-III (in numerical model lies on boundary limit of II and III) and remain constant in other databases and their total average state (Table 5). 
Am. J. Geoscience 1 (1): 7-11, 2010

Table 6: Correlation matrix of landslide databases

\begin{tabular}{llllr}
\hline & A & B & C & \multicolumn{1}{c}{ D } \\
\hline A & 1.0000 & 0.5140 & 0.3154 & 0.1706 \\
B & 0.5140 & 1.0000 & 0.5434 & -0.1640 \\
C & 0.3154 & 0.5434 & 1.0000 & 0.2301 \\
D & 0.1706 & -0.1640 & 0.2301 & 1.0000 \\
\hline
\end{tabular}

The intensity and kind of genetic relation and overlaps of Australian landslide databases is different according to the spearman rank correlation coefficient (based on values of numerical model). The reality of some them can be well explained by following (Table 6):

- Correlation of $\mathrm{R}_{\mathrm{AB}}>\mathrm{R}_{\mathrm{AC}}>\mathrm{R}_{\mathrm{AD}}$ shows data, thematic and spatial relation between databases

- Correlation of $\mathrm{R}_{\mathrm{BC}}>\mathrm{R}_{\mathrm{BA}}>\mathrm{R}_{\mathrm{BD}}$ shows data relation between $\mathrm{A}$ and $\mathrm{B}$, thematic relation of $\mathrm{B}$ and $\mathrm{C}$, but unknown for possible relation between $\mathrm{B}$ and $\mathrm{D}$

- Corelation of $\mathrm{R}_{\mathrm{CB}}>\mathrm{R}_{\mathrm{CA}}>\mathrm{R}_{\mathrm{CD}}$ shows strong data relation between $\mathrm{B}$ and $\mathrm{C}$, medium data relation of $\mathrm{A}$ and $\mathrm{C}$ and low to medium thematic (geotechnical) relation between $\mathrm{C}$ and $\mathrm{D}$

- Correlation of $\mathrm{R}_{\mathrm{DC}}>\mathrm{R}_{\mathrm{DA}}>\mathrm{R}_{\mathrm{DB}}$ shows mainly data

- and spatial relations between $\mathrm{A}$ and $\mathrm{D}$ databases

\section{DISCUSSION}

- According to the results the differences of strengths and weaknesses of the Australian four main landslide databases that mainly affect their practical applicability for hazard management are considerable in spite of genetic relation and data overlaps Thus practical applicability of each database can be characterized as below:

- Database A contains very long statistical time period (more than 160 years) and all over Australia, therefore adequate to the study of landslide temporal and spatial pattern. However, two main gaps in event data $(26.81 \%$ out of 519 landslides) and movement type or class (49.12\%) recording, reduce somewhat its analytical capability and hazard management applicability

- Database B that counted incomparable in the world from the points of study intensity, area coverage and data combination (total risk of 9 main natural hazards on buildings) shows high applicability for landslide risk management

- Database C in spite of high class (IV) of applicability, still needs further detailed studies, spreading of operational circle and coverage of other city centers for better implementation of society and environment management projects

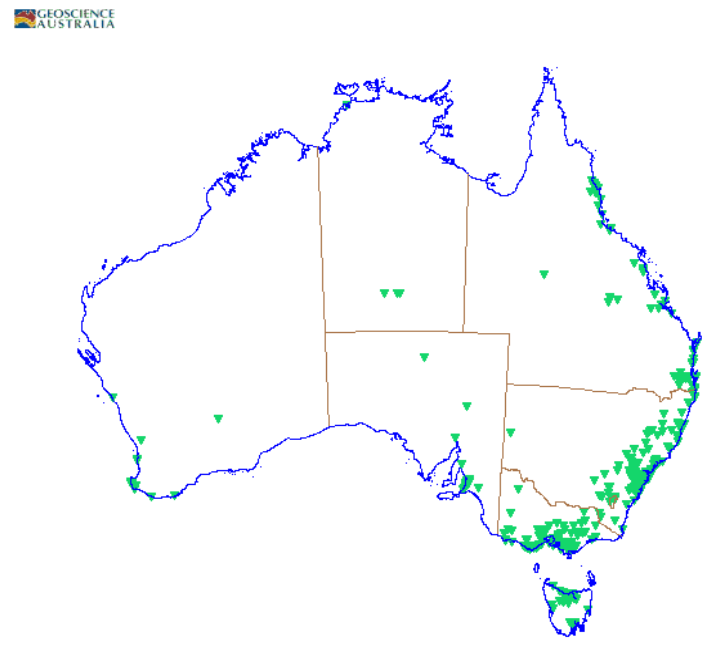

Fig. 2: Australian landslide location map (database)

- Database D, although is main source of geometrical and geotechnical data and affected engineering structures, still needs further presentation of classified data and mapping of landslide hazard management programs

\section{CONCLUSION}

All the available capabilities of Information Technology (IT) in the setup and developing a comprehensive landslide spatial database has not been used yet in Australia as a pioneer country. The current databases need further development and complementary measures especially for compensation of data deficit in event data, geomorphological type, geometrical and geotechnical characteristic and map scale (Fig. 2).

\section{ACKNOWLEDGEMENT}

I thank Dr. R. Drysdale for supervision and assistance of the University of Newcastle, Australia, during my sabbatical leave in 2002.Thanks also due to organizations and persons for providing data sources of this study.

\section{REFERENCES}

Ken, G., J.G. Trevor, L. Marion, S. Greg, 1999. Community risk in Carins, a multi_hazard risk assessment. Aust. J. Emerg. Manage., 14: 25-26. ISSN: 1324-1540

AGSO., 2001. Australian national landside database. http://www.ga.gov.au/oracle/landslid/landsl_online .jsp 
Andrew, K.E., R.J. Blong and Johnson, 1995. Australian landslide database. http://www.ga.gov.au/oracle/landslid/landsl_online .jsp

Barredo, J.I., A. Benavides, J. Hervas and C.J. Van Westen, 2002. Comparing heuristic landslide hazard assessment technique using GIS in the Tirajana basin, ran Cannaria Island, Spain. Int. J. Applied Earth Observ. Geoinform., 2: 9-23. DOI: 10.1016/S0303-2434(00)85022-9

Bontayan, N.C. and I.D. Bishop, 1998. Linking objective and subjective modeling for land use decision making. Landscape Urban Plann., 43: 35-48. DOI: 10.1016/S0169-2046(98)00101-7

CSIRO., 2003. Australia advances, soil cancer, Seri eight.

http:/www.csiro.au/promos/ozadvances/Series8Ca ncer.html

Fell, R., 1995. Theme address-Landslides in Australia. pp: 33.

Geological Survey of Canada, 1999. Landslide disaster databases. pp: 1.

Geoscience Australia, 2002. GEOMET spatial metadata.

http://www.ga.gov.au/bin/htsqr?file=/oracle/geome t/geomet2.htsqr\&datasetno $=2397$

NHRC., 1999. The importance of a good database. http://www.yaldex.com/php_tutorial_3/ch15lev1se c1.html.
NHRC., 2000. Peril Aus I,II (CD-Demo). 6: 23.

Nie, H.F., Diao, S.J., Liu, Ji-X and H. Huan, 2001.The application of remote sensing technique and AHPFuzzy method in comprehensive analysis and assessment for regional stability of Changqing city China. Proceedings of the International Conferences, ISBN: 0-7803-7010-4, pp: 165-170.

Ownegh, M., 2002a. Assessing applicability of Australian landslide databases in hazard management. Proceeding of the 13th International Soil Conservation Organisation Conference, Brisbane, July 2004. pp: 1-5. http://www.tucson.ars.ag.gov/isco/isco13/PAPERS \%20M-Q/OWNEGH\%201.pdf

Ownegh, M., 2002b. Landslide hazard and risk assessment in the southern suburbs of new castle, Australia, sabbatical research project, pp: 100.

Ownegh, M., 2002c. Spatial, temporal and geomorphological pattern of landslides in Australia. sabbatical research report. pp: 69.

Ownegh, M., 2008. Assessing land degradation hazard intensity and management plans using subjective models and the analytical hierarchy process in Gorgan, Iran. Int. J. Sustain. Dev. Plann., 3: 1-11. DOI: 102495/SDP-V3-N4-1-11

Saaty, T.L., 1980. The Analytical Hierarchy ProcessPlanning, Priority Setting, Resource Allocation.1st Edn., McGraw-Hill, Inc., ISBN: 0070543712, pp: 287. 